Research Article

www.jestr.org

\title{
Natural Frequency Sensitivity Analysis of a Road Model Based on the Finite Element Method
}

\author{
WANG Yang ${ }^{1, *}$, Ma Chao ${ }^{2}$, SUI Chuanyi ${ }^{3}$ and SUNG Pontven ${ }^{4}$ \\ ${ }^{l}$ School of Mechanical, Electronic, and Control Engineering, Beijing Jiaotong University, Beijing, 100044, China \\ ${ }^{2}$ School of Civil Engineering, Shijiazhuang Tiedao University, Shijiazhuang, 050043, China \\ ${ }^{3}$ Key Laboratory of Transportation Tunnel Engineering, Ministry of Education, Southwest Jiaotong University, Chengdu, 610031, China \\ ${ }^{4}$ Sun-Moon University, Chung Nam, Korea
}

Received 11 April 2014; Accepted 23 July 2014

\begin{abstract}
The vibration caused by moving loads gradually destroys the road, negatively affecting the performance of the road structure. Therefore, a study on the natural frequency of a road structure is necessary for a dynamic analysis of the road. In this study, the natural frequency of an expressway was calculated based on the finite element method with the help of the ABAQUS software. Results indicated that the natural frequencies of the first to the fifth orders of the road were 4.016, $4.271,4.361,4.431$ and $4.956 \mathrm{~Hz}$, respectively. Further study indicated that natural frequency was sensitive to model size, such that the former decreased when the latter increased. The result of natural frequency was highly sensitive to length (along the driving direction) and thickness. A suitable model size should be selected to ensure a reliable and accurate calculation result of natural frequency. The suitable model size should be $3.75 \mathrm{~m}$ wide (one lane), $30 \mathrm{~m}$ long (along the drive direction), and $10 \mathrm{~m}$ thick. The calculation result of natural frequency was also highly sensitive to boundary conditions. Therefore, rational boundary constraints should be determined to obtain accurate results. The calculation results of natural frequency differed slightly with the variations in contact conditions between layers. Fully continuous contact conditions between layers should be selected for the road model. The natural frequency was not sensitive to the horizontal alignment parameter of the road, remaining constant when the horizontal radius changed from $\infty$ to $1000 \mathrm{~m}$. Thus, the effect of the horizontal alignment parameter on the natural frequency can be ignored. Furthermore, the natural frequency was not sensitive to the material density of the road except that of the sub-grade material. No obvious change was observed from the calculation result of natural frequency when the material Poisson's ratio varied.
\end{abstract}

Keywords: Finite Element Method, Expressway, Road Structure, Natural Frequency

\section{Introduction}

Various loadings with different frequencies occur with the increase of daily traffic and vehicle speed. Resonance occurs if the loading frequency is close to the natural frequency of the road structure, and the dynamic damage to the pavement is accelerated [1]. Therefore, a study on the natural frequency of the road structure is necessary to further study on the dynamic response of the road under different loading frequencies.

Few studies on road natural frequency have been conducted. Yoo et al reported that resonance might occur when the vehicle speed is high and the temperature is low because the loading frequency and the road natural frequency are very close under such conditions [2]. Yuan et al studied the natural frequency of asphalt pavement by employing the finite element method, and found that the natural frequencies of the first order were 2.6 and $6.4 \mathrm{~Hz}$, and those of the second order were 4.6 and $12.3 \mathrm{~Hz}$, before and after cracks occurred in the pavement, respectively [3].

*E-mail address: jtxwy@163.com

ISSN: 1791-2377 @ 2014 Kavala Institute of Technology. All rights reserved.
Using FEM, Zhang et al calculated the natural frequency of the road to be $17.023 \mathrm{~Hz}$. They further concluded that no resonance occurred on the pavement [4]. Sun et al determined that the natural frequency of urban road ranged from $5 \mathrm{~Hz}$ to $15 \mathrm{~Hz}$ during their study of the environmental effect of city road traffic [5]. In their study of the relationship between the dynamic response of asphalt pavement and the distance of the vibration source, Yuan et al argued that the natural frequency of the highway ranged from $10 \mathrm{~Hz}$ to $60 \mathrm{~Hz}$ [6]. Zhao et al analyzed the natural frequency of semi-rigid pavement by employing both the FEM and the experimental method, concluding that natural frequency of such pavement ranged from $10 \mathrm{~Hz}$ to $20 \mathrm{~Hz}$ [7].

The FEM is generally used for the natural frequency analysis of road. However, the results are diverse. Thus, conducting further studies is necessary. This paper aims to study the natural frequency sensitivity of the road to discover how the parameters of the FEM affect the calculation result of natural frequency. This study may serve as a reference in developing a finite element model for the structural dynamic response analysis of roads. 


\section{Fundamental theory of Natural Frequency Analysis for Structures}

The basic aim of natural frequency analysis based on the FEM is to identify the eigenvalues of a motion equation with finite freedom without loading. The damping of the structure can be ignored because it only slightly affects the results. The free vibration equation of the structure without damping can be illustrated in matrix form given by

$[M]\{\ddot{u}\}+[K]\{u\}=\{0\}$

where $[M]$ is the mass matrix, $[K]$ is the stiffness matrix, and $\{u\}$ is the displacement of the system.

For a linear structure system, $[M]$ and $[K]$ are both real symmetric matrixes. The equation has a solution in the form of simple harmonic motion given by

$$
u=\Phi \mathrm{e}^{i \omega_{n} t}
$$

where $\Phi$ is the amplitude of $u$, and $\omega$ is the angular frequency of the simple harmonic motion.

When Equation (2) is integrated into Equation (1), Equation (3) can be obtained as

$$
\left[K-\omega_{n}^{2} M\right]\{\Phi\} \mathrm{e}^{i \omega_{n} t}=\{0\}
$$

Given that Equation (3) is workable at any time, the $t$ function part can be removed. Then, Equation (4) can be obtained as

$$
\left[K-\omega_{n}^{2} M\right]\{\Phi\}=\{0\}
$$

Equation (4) is a typical equation with real eigenvalues. If the value of its coefficient determinant is zero, as shown in Equation (5), then $\Phi$ has a nonzero solution expressed as

$$
\left|K-\omega_{n}^{2} M\right|=0
$$

A set of discrete roots $\omega_{i}(i=1,2, \ldots, n)$ can be obtained based on Equation (5). When these solutions are integrated into Equation (4), $\Phi_{i}(i=1,2, \ldots, n)$ can be obtained accordingly, where $\omega_{i}$ is the $i$ th eigenvalue of the structure (i.e., natural frequency) and $\Phi_{i}$ is the $i$ th eigenvector (i.e., vibration mode) [8].

\section{Preliminary Analysis of the Natural Frequency of a Typical Road Structure Based on the FEM}

The size of the finite element model is determined by a typical semi-rigid pavement of an expressway with six lanes. Half of the cross section is studied to simplify the model. The top width of the model is $17.25 \mathrm{~m}$ ( $X$-axis of Figure 1$)$, which is composed of a grass median $(1.5 \mathrm{~m})$, a left curb $(0.75 \mathrm{~m})$, three lanes $(11.25 \mathrm{~m})$, a hard shoulder $(3 \mathrm{~m})$, and an earth shoulder $(0.75 \mathrm{~m})$. The slope of the cross section is $1: 1$, and the slope height is $2 \mathrm{~m}$. The thickness of the model is $10 \mathrm{~m}$ ( $Y$-axis of Figure 1), and the length of the model is $30 \mathrm{~m}$ along the drive lane ( $Z$-axis of Figure 1$)$. The structure and material parameters of the road based on a previous work [7] are shown in Table 1.

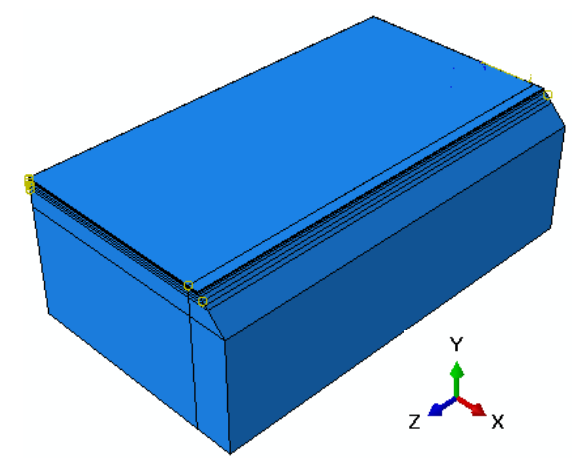

Fig.1. Road model

Tab. 1. Pavement structure and material parameters

\begin{tabular}{ccccc}
\hline Road Structure & $\begin{array}{c}\text { Thickness } \\
/ \mathrm{cm}\end{array}$ & $\begin{array}{c}\text { Density } \\
/ \mathrm{kg} \cdot \mathrm{m}^{-3}\end{array}$ & $\begin{array}{c}\text { Modulus } \\
/ \mathrm{MPa}\end{array}$ & Poisson's Ratio \\
\hline Surface Course & 4 & 2500 & 1600 & 0.35 \\
Binder Course & 16 & 2450 & 1600 & 0.35 \\
Base Course & 36 & 2400 & 1600 & 0.25 \\
Sub-Base & 20 & 1800 & 1400 & 0.20 \\
Sub-Grade & - & 1850 & 40 & 0.40 \\
\hline
\end{tabular}

The basic hypothesis is presented as follows: (1) each layer is anisotropic linearly elastic material, (2) the displacement in all directions is 0 at the bottom of the model, (3) the horizontal displacements in the $Y-Z$ and the $X-Y$ planes are 0 , and the slope is free, and (4) the contact conditions between the layers are fully continuous [9], [10].

The natural frequency is calculated with the help of the ABAQUS software. The first- to fifth-order vibration modes are shown in Figure 2. The natural frequencies of the five orders are 4.016, 4.271, 4.361, 4.431 and $4.956 \mathrm{~Hz}$, respectively. They are close to the results listed in [3].

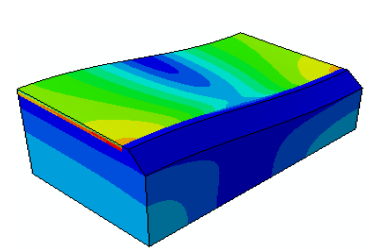

a) first order

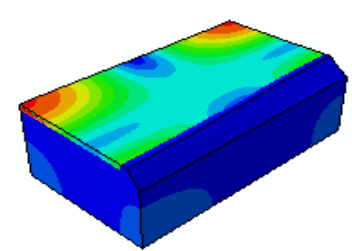

c) third order

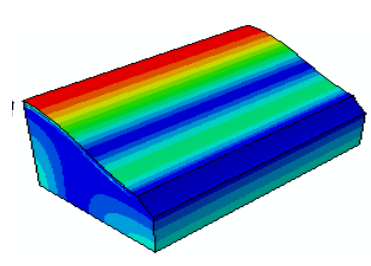

b) second order

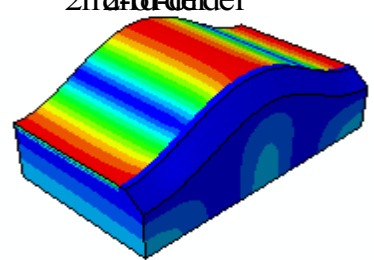

d) fourth order

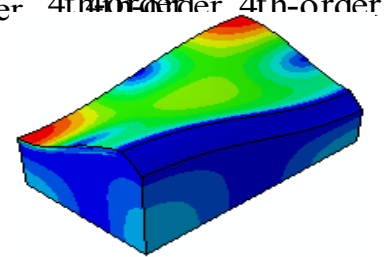

e) fifth order 
Fig.2. First- to fifth-order vibration modes

\section{Analysis of Natural Frequency Sensitivity to the Model Size}

Next, natural frequency sensitivity to the model size (i.e., length, width, and thickness) is analyzed. Sixteen model cases with different sizes are developed. The size of each case is listed in Table 2.

Tab. 2. Sixteen model cases with different sizes (m)

\begin{tabular}{|c|c|c|c|}
\hline Cases & Width ( $X$-axis) & Length ( $Z$-axis) & Thickness ( $Y$-axis) \\
\hline 1 & 3.75 (1 lane) & 30 & 5 \\
\hline 2 & 7.50 (2 lanes) & 30 & 5 \\
\hline 3 & 11.25 (3 lanes) & 30 & 5 \\
\hline 4 & 3.75 & 10 & 10 \\
\hline 5 & 3.75 & 15 & 10 \\
\hline 6 & 3.75 & 20 & 10 \\
\hline 7 & 3.75 & 25 & 10 \\
\hline 8 & 3.75 & 30 & 10 \\
\hline 9 & 3.75 & 30 & 3 \\
\hline 10 & 3.75 & 30 & 4 \\
\hline 11 & 3.75 & 30 & 5 \\
\hline 12 & 3.75 & 30 & 6 \\
\hline 13 & 3.75 & 30 & 7 \\
\hline 14 & 3.75 & 30 & 8 \\
\hline 15 & 3.75 & 30 & 9 \\
\hline 16 & 3.75 & 30 & 10 \\
\hline
\end{tabular}

Cases 1 to 3 have different widths (X-axis), while the length and thickness remain constant. The calculation results of the natural frequencies of cases 1 to 3 are plotted in Figure 3. The results clearly show the natural frequency sensitivity to width.

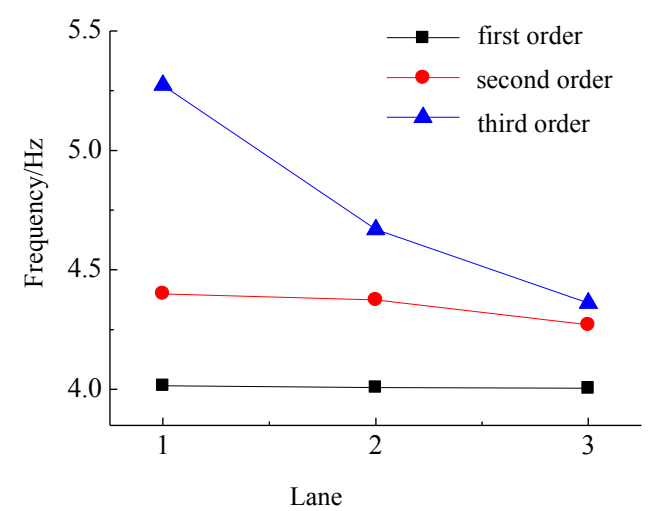

Fig.3. Natural frequency-lane curve

Figure 3 shows that the natural frequencies of all three orders range from $4 \mathrm{~Hz}$ to $5.5 \mathrm{~Hz}$. Increasing the width causes the natural frequency values to decrease. The decline ratio and magnitude of the natural frequencies of the first and second orders are much smaller than those of the third order when the width increases from one to three lanes. The values of the first- and second-order natural frequencies are decreased by $0.27 \%$ and $2.9 \%$, respectively, whereas the value of the third order is decreased by $17.3 \%$.

Cases 4 to 8 have different lengths (Z-axis), while the width and thickness remain constant. The calculation results of the natural frequency of cases 4 to 8 are plotted in Figure 4. The results clearly show the natural frequency sensitivity to length.

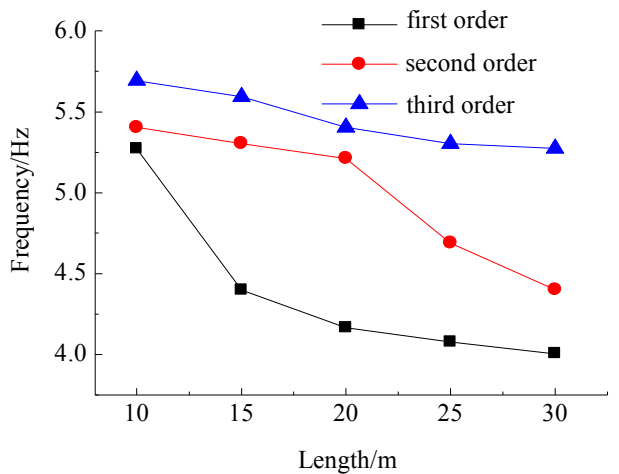

Fig.4. Natural frequency-length curve

The frequencies of all three orders generally decrease if the length increases (Figure 4). Specifically, the decline rates of the first- and third-order frequencies decrease when the length increases from $20 \mathrm{~m}$ to $25 \mathrm{~m}$. The decline rate of a second-order frequency decreases when the length increases from $25 \mathrm{~m}$ to $30 \mathrm{~m}$. Furthermore, the frequency of each order levels off when the length is greater than $30 \mathrm{~m}$.

Cases 9 to 16 have different thicknesses (Y-axis), while the width and length remain constant. The calculation results of the natural frequency of cases 9 to 16 are plotted in Figure 5 . The results clearly show the natural frequency sensitivity to thickness.

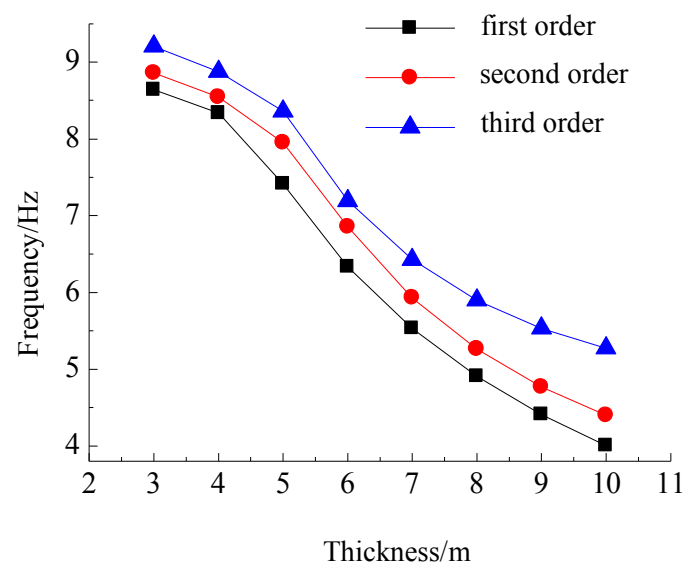

Fig.5. Natural frequency-thickness curve

The frequencies generally decrease if the thickness increases (Figure 5). The natural frequencies of the first, second, and third orders are decreased by $54.6 \%, 54.8 \%$ and $40.8 \%$, respectively, when the thickness increases from $3 \mathrm{~m}$ to $10 \mathrm{~m}$

To sum up, natural frequency is highly sensitive to the values of length and thickness. Therefore, a suitable width and length should be designed. Hence, a single-lane, length of $30 \mathrm{~m}$, and thickness of $10 \mathrm{~m}$ are reasonable values according to the aforementioned results. 


\section{Analysis of Natural Frequency Sensitivity to Boundary Constraints}

Four boundary constraint cases (Table 3 ) are designed for the study of natural frequency sensitivity to boundary constraints. The size of the model is $3.75 \mathrm{~m}$ (single lane) wide, $10 \mathrm{~m}$ thick, and $30 \mathrm{~m}$ long. The natural frequencies from the first to the third order of each case are shown in Table 4.

Tab. 3. Boundary constraints

\begin{tabular}{ll}
\hline Case & \multicolumn{1}{c}{ Boundary Constraints } \\
\hline 1 & $\begin{array}{l}\text { The bottom is free, and the vertical displacements of the } X-Y \\
\text { and } Y-Z \text { planes are constrained. }\end{array}$ \\
2 & $\begin{array}{l}\text { The bottom is fixed, and the vertical displacements of the } X-Y \\
\text { and } Y-Z \text { planes are constrained. }\end{array}$ \\
3 & $\begin{array}{l}\text { The bottom is free, and no displacement is permitted in any } \\
\text { direction of the } X-Y \text { and } Y-Z \text { planes. }\end{array}$ \\
4 & $\begin{array}{l}\text { The bottom is fixed, and no displacement is permitted in any } \\
\text { direction of the } X-Y \text { and } Y-Z \text { planes. }\end{array}$
\end{tabular}

Tab. 4. Frequencies under different boundary conditions $(\mathrm{Hz})$

\begin{tabular}{llll}
\hline \multirow{2}{*}{ Case } & \multicolumn{3}{c}{ Natural Frequency } \\
\cline { 2 - 4 } & \multicolumn{1}{c}{ First Order } & \multicolumn{1}{c}{ Second Order } & Third Order \\
\hline 1 & 2.621 & 3.651 & 4.879 \\
2 & 4.005 & 4.400 & 5.273 \\
3 & 5.545 & 6.204 & 7.077 \\
4 & 6.154 & 7.161 & 7.184 \\
\hline
\end{tabular}

Table 4 shows that the natural frequencies of cases 1 to 4 gradually increase. This result indicates that the more the boundary constraints, the higher the natural frequency.

\section{Analysis of Natural Frequency Sensitivity to Contact Conditions between Layers}

Three contact conditions are developed for the study of natural frequency sensitivity to contact conditions between layers. The three contact conditions are fully slip, frictionally slip, and fully continuous. The size of the model is $3.75 \mathrm{~m}$ (single lane) wide, $10 \mathrm{~m}$ thick, and $30 \mathrm{~m}$ long. The calculation results of the natural frequency of each case are shown in Figure 6.

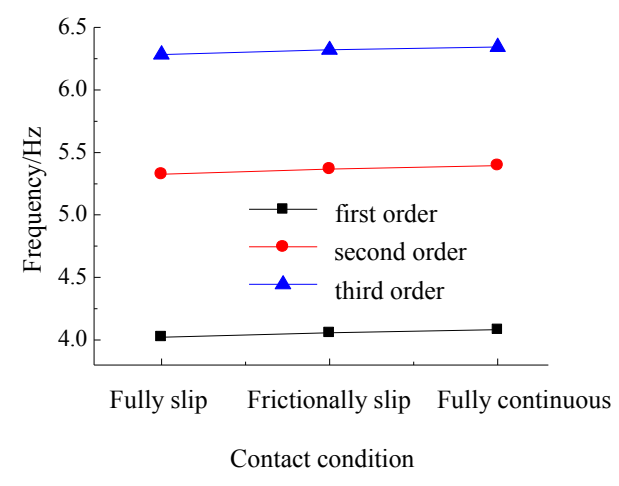

Fig.6. Curve of the natural frequency-contact condition

The natural frequency of each order slightly varies when the contact condition varies (Figure 6). When the contact condition changes from fully slip to fully continuous, the frequency is only increased by $1.5 \%, 0.95 \%$, and $0.97 \%$ for the first, second and third orders, respectively. Therefore, the effect of contact condition on the calculation result can be ignored given the small affecting magnitude. However, fully continuous contact should be designed for the dynamic response analysis of road structures, considering the strong lateral constraint between different layers.

\section{Analysis of Natural Frequency Sensitivity to Horizontal Alignment Parameters}

A circular curve model case with a $1000 \mathrm{~m}$ radius and $6 \%$ cant rate is developed in the study of natural sensitivity to horizontal alignment parameters. The model size is $3.75 \mathrm{~m}$ (single lane) wide, $10 \mathrm{~m}$ thick, and $30 \mathrm{~m}$ long (outside arc). The natural frequency of the circular model case is calculated, and the comparison of natural frequencies for straight versus circular curve sections is shown in Table 5.

Tab. 5. Comparison of natural frequencies of straight versus circular curve sections

\begin{tabular}{llll}
\hline \multirow{2}{*}{ Horizontal Alignment } & \multicolumn{3}{c}{ Natural Frequency } \\
\cline { 2 - 4 } & First Order & Second Order & Third Order \\
\hline $\begin{array}{l}\text { Circular curve } \\
(R=1000 \mathrm{~m})\end{array}$ & 3.998 & 4.398 & 5.256 \\
\begin{tabular}{l} 
Straight $(R=\infty)$ \\
\hline
\end{tabular} & 4.005 & 4.400 & 5.273 \\
\hline
\end{tabular}

Table 5 shows that the natural frequency of each order of the straight model is slightly greater than that of the circular curve model (increased by $0.17 \%, 0.04 \%$, and $1.7 \%$ for the first, second and third orders, respectively). Therefore, the natural frequency is not sensitive to the horizontal alignment parameters. However, sensitivity should be considered for the dynamic response analysis of road structures, considering the complex loading applied on the pavement in the curve section.

\section{Analysis of Natural Frequency Sensitivity to Material Density}

The natural frequency sensitivity to material density is analyzed in this section. The size of the model is $3.75 \mathrm{~m}$ (single lane) wide, $10 \mathrm{~m}$ thick, and $30 \mathrm{~m}$ long. The assumptions made are as follows: the surface and binder courses are made of asphalt mixture, the base course and sub-base are made of stabilized soil, and the sub-grade is made of soil. Five density cases are designed for each material. The results of natural frequency of the first, second, and third orders are shown in Figures 7, 8 and 9, respectively.

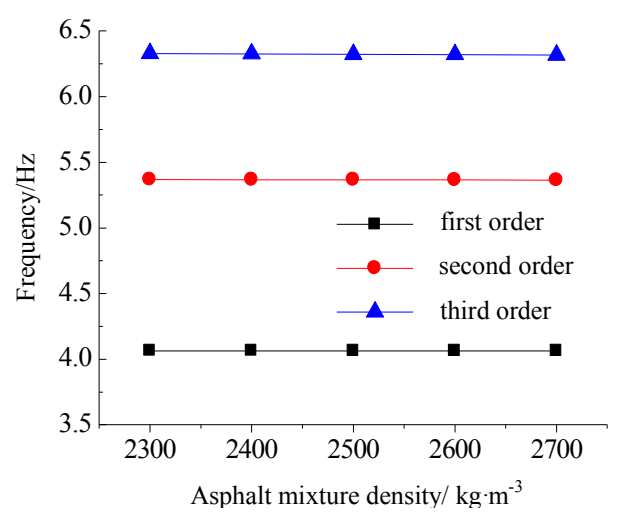


Fig.7. Curve of natural frequency-asphalt mixture density

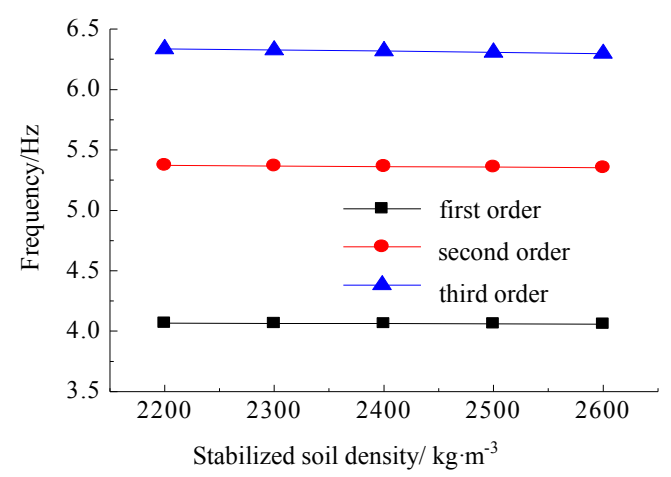

Fig.8. Curve of natural frequency-stabilized soil density

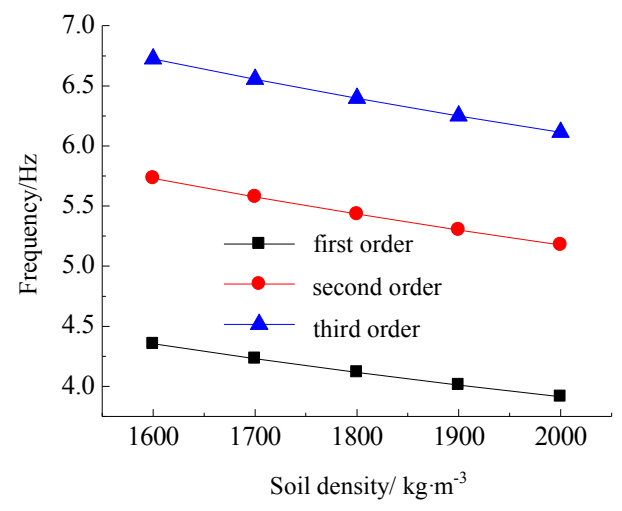

Fig.9. Curve of natural frequency-soil density

Figures 7 to 9 show that the natural frequency is slightly sensitive to the densities of the asphalt mixture and stabilized soil, respectively. This result may be attributed to the fact that the thickness of these layers is too small to have an obvious effect on the total mass of the structure. As a result, it is difficult to affect the natural frequency of the whole structure. However, the natural frequency is highly sensitive to the density of soil, that is, it sharply decreases when the soil density increases. The frequencies of the first, second, and third orders decrease by $10.1 \%, 9.6 \%$ and $9.1 \%$, respectively, when the density increases from $1600 \mathrm{~kg} \cdot \mathrm{m}^{-3}$ to $2000 \mathrm{~kg} \cdot \mathrm{m}^{-3}$. This may be attributed to the fact that the mass of the sub-grade is sufficiently large to have a great effect on the total mass of the structure. Consequently, the mass has an obvious effect on the natural frequency of the structure.

\section{Analysis of Natural Frequency Sensitivity to the Poisson's Ratio of the Material}

The natural frequency sensitivity to the Poisson's ratio of the material is studied in this section. The model size is $3.75 \mathrm{~m}$ (single lane) wide, $10 \mathrm{~m}$ thick, and $30 \mathrm{~m}$ long. Four Poisson's ratio cases are designed for each material (i.e., asphalt mixture, stabilized soil, and soil). The results of natural frequency of the first, second, and third orders for different cases are shown in Figures 10, 11 and 12, respectively.

Figures 10 to 12 show that no obvious change can be observed for the natural frequency when the Poisson's ratio of the material varies.

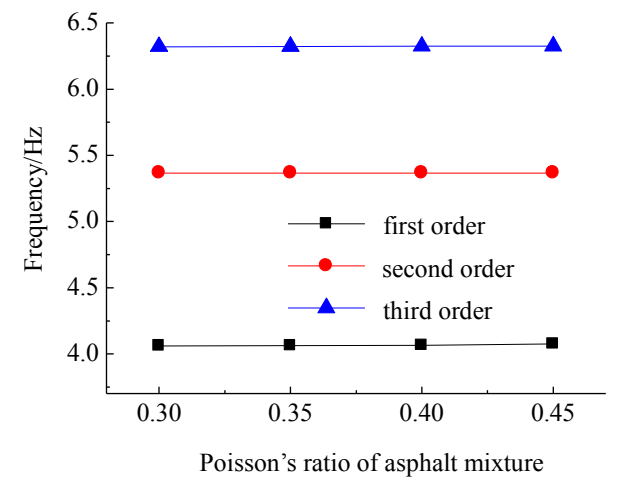

Fig.10. Curve of natural frequency-Poisson's ratio of asphalt mixture

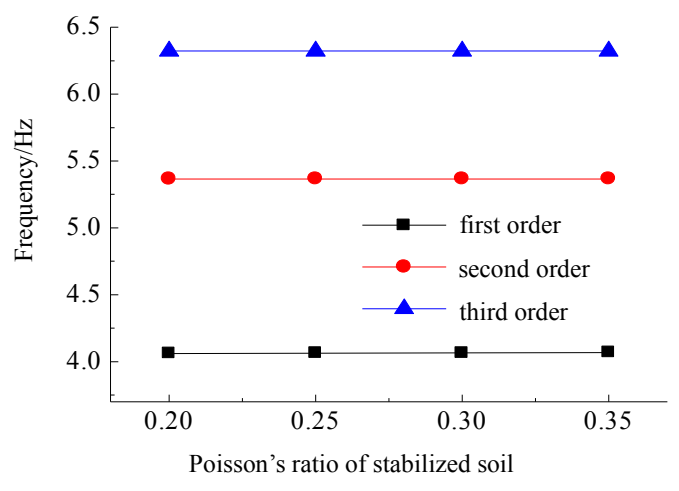

Fig.11. Curve of natural frequency-Poisson's ratio of stabilized soil

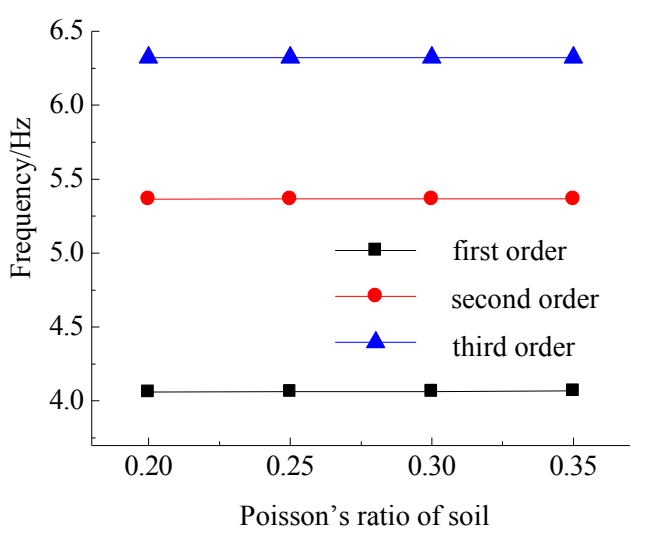

Fig.12. Curve of natural frequency-Poisson's ratio of soil

\section{Conclusions}

The natural frequency of a road structure with different parameters is calculated, and the natural frequency sensitivity to the parameters is analyzed in this study using the ABAQUS software. Several conclusions are obtained.

First, the calculation result of natural frequency is sensitive to the model size, that is, frequency decreases when the model size increases. A suitable model size should be designed for the dynamic characteristic analysis of road structures to obtain reliable and accurate results. The following parameters are reasonable for the model size: single lane, length of $30 \mathrm{~m}$, and thickness of $10 \mathrm{~m}$.

Second, the calculation result of natural frequency is highly sensitive to the boundary constraints. Thus, rational boundary constraints should be designed for the dynamic 
characteristic analysis of road structures, in order to obtain reliable and accurate results.

Third, the calculation result of natural frequency is not nearly as sensitive to the contact conditions between layers. However, fully continuous contact should be designed for the dynamic response analysis of road structures, considering the strong lateral constraint between different layers.

Fourth, natural frequency is not sensitive to the horizontal alignment parameters. Hence, the effect of horizontal alignment on the natural frequency can be ignored. However, sensitivity should be considered for the dynamic response analysis of road structures, considering the complex loading applied to the pavement in the curve section.

Finally, natural frequency is not sensitive to the material density of the road, except that of the sub-grade material. No obvious change is observed from the calculation result of natural frequency when the Poisson's ratio of the material varies.

\section{Acknowledgments}

The authors would like to thank the National Natural Science Foundation of China for their financial support (Grant Nos. 11302138, 11172183, and 11102121).

\section{References}

1. Chatti K. and Lee D., "Development of a preventive maintenance strategy for minimizing roughness-related pavement damage", Transportation Research Record: Journal of the Transportation Research Board, (1769), 2001, pp. 39-45.

2. Yoo P.J. and Al-Qadi I.L., "Effect of transient dynamic loading on flexible pavements", Transportation Research Record: Journal of the Transportation Research Board, (1990), 2007, pp. 129-140.

3. Yuan H.-K., Song X.-P. and Qian Z.-D., "Analysis of the dynamic characteristics of asphalt pavement with cracks", Journal of Transportation Engineering and Information, 5(1), 2007, pp. 108113.

4. Zhang Z.-Q., Fu H.-B. and Jin J., "Analysis of the dynamic characteristics of asphalt pavement under heavy loads", Journal of Beijing University of Technology, 33(7), 2007, pp. 693-696.

5. Sun Y.-M., Huang L.-L. and Yu J.-Q., "The environmental effect evoked by city road traffic", Journal of Shanghai Institute of Technology, 6(4), 2006, pp. 233-235.
6. Yuan X.-M., Zhang Y.-H. and Zuo P.-F., et al, "Field test and analysis of vibration induced by traffic", Soil Engineering and Foundation, 21(2), 2007, pp. 73-75.

7. Zhao H.-D. and Liang Y.-H., "Natural frequency of asphalt pavement via finite element analysis", Journal of Transport Information and Safety, 30(2), 2012, pp. 10-15.

8. Thomson W. T. and Dahleh M. D., "Theory of Vibration with Applications", 5th Edition, Prentice Hall: New Jersey, 1997.

9. Saad B., Mitri H. and Poorooshasb H., "Three-dimensional dynamic analysis of flexible conventional pavement foundation", Journal of Transportation Engineering, 131(6), 2005, pp. 460-469.

10. Johnson D.J., "Investigation of performance of flexible pavement systems under moving loads using finite element analyses". Rowan University: Glassboro. 2008. 